\title{
Dissection of an old protein reveals a novel application: domain D of Staphylococcus aureus Protein A (sSpAD) as a secretion - tag
}

Thomas Heel ${ }^{1,2,3}$, Michael Paal ${ }^{1,2,3}$, Rainer Schneider ${ }^{1,2,3}$, Bernhard Auer ${ }^{1,2,3^{*}}$

\begin{abstract}
Background: Escherichia coli as a frequently utilized host organism for recombinant protein production offers different cellular locations with distinct qualities. The periplasmic space is often favored for the production of complex proteins due to enhanced disulfide bond formation, increased target product stability and simplified downstream processing. To direct proteins to the periplasmic space rather small proteinaceus tags that can be used for affinity purification would be advantageous.

Results: We discovered that domain D of the Staphylococcus aureus protein A was sufficient for the secretion of various target proteins into the periplasmic space of E. coli. Our experiments indicated the Sec pathway as the mode of secretion, although $\mathrm{N}$-terminal processing was not observed. Furthermore, the solubility of recombinant fusion proteins was improved for proteins prone to aggregation.

The tag allowed a straightforward affinity purification of recombinant fusion protein via an IgG column, which was exemplified for the target protein human superoxide dismutase 1 (SOD).

Conclusions: In this work we present a new secretion tag that combines several advantages for the production of recombinant proteins in E. coli. Domain D of S. aureus protein A protects the protein of interest against N-terminal degradation, increases target protein solubility and enables a straight-forward purification of the recombinant protein using of lgG columns.
\end{abstract}

\section{Background}

Due to the simple handling, inexpensive fast high-density cultivation and well-known genetics [1,2], E. coli remains an attractive host for the production of recombinant proteins, even though more complex proteins with posttranslational modifications, such as glycosylation patterns require alternative host systems $[3,4]$. Depending on the characteristics of the target protein, E. coli offers different compartments to meet the requirements for successful expression and purification. Cytoplasmic expression offers high yields of soluble product [5], but the purification from the cell lysate can be complex and costly. High level cytoplasmic overexpression may lead to the formation of inclusion bodies (IB).

\footnotetext{
* Correspondence: bernhard.auer@uibk.ac.at

${ }^{1}$ Austrian Center of Industrial Biotechnology (ACIB), TU Graz, Petersgasse 14 A-8010 Graz, Austria

Full list of author information is available at the end of the article
}

These protein aggregates simplify the purification but make in vitro refolding necessary [6,7].

In order to make purification easier, protect the target from degradation, (which is especially a problem with low molecular weight molecules [8]) or increase the chance of proper folding, the secretion of the target protein into the periplasm or the culture medium has proven to be a strong alternative $[9,10]$. Translocation of proteins across the inner membrane requires a signal peptide. However, the presence of a signal sequence alone does not ensure secretion into the periplasmic space $[11,12]$. Thus, a larger secretion moiety can be linked to the target gene. It has been shown that the Staphylococcus aureus Protein A (SpA) secretion signal combined with miscellaneous Protein A sub-domains directs heterologous proteins into the periplasm or even to the culture supernatant [13]. In addition to promoting protein translocation to the periplasm these domains
Ciomed Central

C 2010 Heel et al; licensee BioMed Central Ltd. This is an Open Access article distributed under the terms of the Creative Commons Attribution License (http://creativecommons.org/licenses/by/2.0), which permits unrestricted use, distribution, and reproduction in any medium, provided the original work is properly cited. 
have been shown to improve folding of the target protein and to protect against N-terminal degradation [14].

In the present work we show that domain D of SpA expressed from a synthetic codon optimized gene (sSpAD) is sufficient for the secretion of recombinant proteins. Furthermore we propose the Sec pathway mediating secretion and demonstrate the possibility of a straightforward one step expression and purification system.

\section{Results and discussion}

Preliminary experiments with the swine fever virus autoprotease $\mathrm{N}^{\text {pro }}$ EDDIE [7] showed that protein solubility was drastically increased with a C-terminal sSpAD extension, whereas $\mathrm{N}^{\text {pro }}$ EDDIE fusion proteins with other tags were deposited as insoluble aggregates within the cytoplasm $[7,15]$. Due to the characteristics of protein $A$ as a surface protein it was assumed that an $\mathrm{N}$-terminal sSpAD tag might facilitate periplasmic secretion. Therefore a construct with an sSpAD tag upstream of the autoprotease was cloned and this fusion protein was expressed under control of the weak lacUV5 promoter in E. coli. As shown in Figure 1 the tag enhanced the solubility of the aggregate forming protein $\mathrm{N}^{\mathrm{pro}} \mathrm{ED}$ DIE-pep6His by a factor of three. The soluble fraction of human superoxide dismutase 1 (SOD), which is a highly soluble protein per se (95\%), was enhanced only by three percent. However, solubility of GFPmut3.1 even decreased as a fusion with sSpAD. This can be explained by the observation that E.coli did not tolerate considerable amounts of GFPmut3.1 in the cytoplasm. We found the main portion of GFPmut3.1 either in the periplasm or in inclusion bodies (data not shown). In

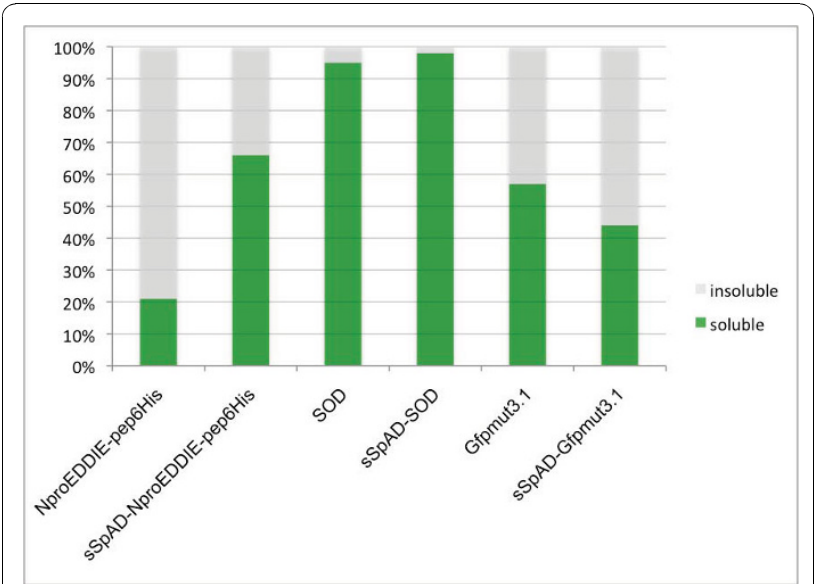

Figure 1 Comparison of the protein solubility with and without

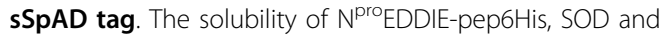
GFPmut3.1 with and without SSpAD tag, expressed under the control of a LacUV5 promoter, was determined under defined cultivation conditions. fusion with sSpAD the expression rate was increased even further. The additional amount of protein was mostly deposited in inclusion bodies and only a minor part exported to the periplasm (data not shown). This led to a slightly increased export but to an overall reduced solubility.

Subsequent isolation of the periplasmic fraction by osmotic shock treatment revealed the ability of the sSpAD moiety to mediate secretion. The different cell compartments were isolated according to the manual published by Paal et al. [16]. There are two main secretion pathways, known to mediate protein translocation across the inner membrane [17]. A number of specific inhibitors were used to distinguish between passive diffusion and active secretion by means of the Sec or Tat pathway.

\section{Identification of the secretion pathway}

In order to identify the secretion pathway the fusion construct sSpAD-N ${ }^{\text {pro }}$ EDDIE-pep6His was expressed under control of the strong T5 promoter. This promoter enabled the expression of detectable protein amounts in the presence of toxins, such as carbonyl cyanide mchlorphenylhydrazone (CCCP) within short incubation times in different host strains.

Several secretion pathways can be analyzed using diverse protonophores and knockout strains. CCCP has the ability to specifically inhibit all proton motive force driven pathways in $E$. coli $[18,19]$. To test if sSpAD secretion was driven by any of these pathways the recombinant fusion protein was expressed and cultivated in the presence of CCCP. As shown in Figure 2A, CCCP inhibited secretion into the periplasm. The slight signal in the periplasmic fraction could be considered as an artefact because minor expression of the recombinant protein occurs even before induction and addition of CCCP. Thus the signal in the periplasmic fraction was most likely due to secretion before inhibition of the given secretion pathway. Immuno blots against GroEL were performed to confirm the integrity of the spheroplasts. GroEL is a cytoplasmic chaperone, which was not found in the periplasm (Figure 2B). Anti Maltose binding protein $\mathrm{E}$ (MalE) immuno blots were performed to verify the isolated fractions as periplasm of $E$. coli cells (Figure 2C).

DADE is a MC4100 derived Tat knock-out strain lacking a functional Tat pathway [20]. This strain was used to show if sSpAD secretion was Tat dependent. Subcellular fractions showed that Tat knockout had no effect on the secretion capacity of the fusion protein shown in Figure 2A. Therefore, the involvement of the Tat pathway in the secretion process was excluded.

Sodium-azide is a strong inhibitor of the ATPase SecA and therefore has the ability to specifically inhibit the 


Figure 2 Secretion competence of an sSpAD fusion protein. A: The sSpAD tag reacts with most secondary antibodies. Therefore sSpAD
fusion proteins were detected on the same membrane as GroEL immuno blots. For the sSpAD immuno blots a secondary antibody was used
which reacts against sSpAD. B: Anti GroEL immuno blots of the isolated fractions were performed to confirm that the periplasmic fractions
obtained via osmolysis, were not contaminated with cytoplasmic proteins. C: Anti MalE immuno blots were performed to confirm that the
isolated fractions correspond with the periplasm as the Maltose Binding Protein $\mathrm{E}$ is a periplasmic protein. -l: whole cell sample without
induction of recombinant protein expression, +l: whole cell samples with induction of recombinant protein expression, P periplasmic fraction,
C: cytoplasmic fraction; CCCP: carbonyl cyanide m-chlorophenylhydrazone, BL21(DE3): host strain, DADE: $\triangle$ tataBCD $\Delta$ tatE host strain derived from
MC4100.

Sec translocation [21] in E. coli. Preliminary experiments with sodium-azide indicated an inhibition of translocation, although, recombinant expression artefacts were observed in the periplasmic fraction due to the osmotic shock procedure. Therefore, experiments were performed in parallel, using the target protein with and without the secretion tag to determine the background. The densitometric comparison of the amount of recombinant protein with and without SSpAD-tag in the periplasmic fraction confirmed that sodium-azide inhibited secretion. Continuative experiments were performed with the SecE knock-out strain CM124 [21]. As the Sec pathway is crucial for cell viability, SecE was complemented via expression from an L-arabinose induced plasmid. Over-night (including L-arabinose) culture was split, diluted and subsequently cultivated with and without L-arabinose. After dilution the culture without Larabinose should have no functional Sec pathway. As shown in Figure 3 the knockout of the Sec pathway inhibited secretion of the target protein. These experiments led to the assumption that the Sec pathway seems to be involved in some way in the export of sSpAD fusion proteins to the periplasm; however the SignalP 3.0 prediction tool http://www.cbs.dtu.dk/services/SignalP did not reveal a canonical Sec secretion signal. The recent advances in genomics and proteomics revealed numerous extracellular proteins lacking defined secretion signals [22], often combining functions within the cytoplasm and in the extracellular environment [23]. Possibly, the elevated levels of expression of the sSpAD tagged proteins could lead to an emergency mechanism, which exports proteins through the Sec channel without using a signal sequence.

\section{Quantification of the secretion capacity}

The fusions of sSpAD to the pestiviral autoprotease $\mathrm{N}^{\text {pro }}$ EDDIE-pep6His, to green fluorescent protein GFPmut3.1 and to human superoxide dismutase 1 (SOD) produced by shaking flask cultivation were quantified by densitometry of SDS-PAGE as described in Methods. Since the fusion tag was not cleaved after secretion, it was not possible to distinguish easily between secreted and non-secreted proteins. Preliminary experiments showed that overexpressed proteins lacking export signals were detected in the periplasmic fraction. Therefore all recombinant proteins were expressed with and without SSpAD tag and the periplasmic fractions of all samples were isolated. Subsequently, the concentrations of the target proteins in the periplasm of all samples were measured and the amount found in the

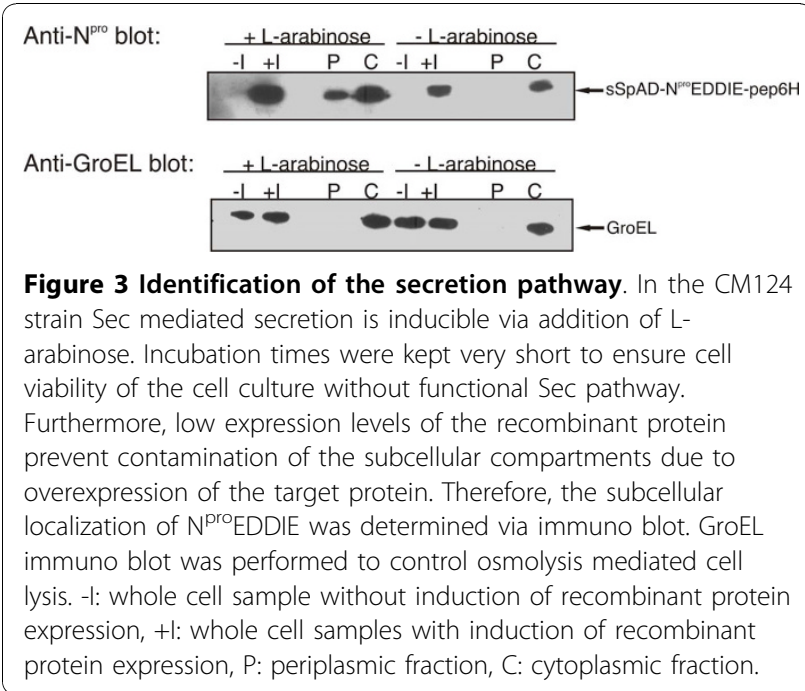


Table 1 Secretion capacity of the fusion proteins

\begin{tabular}{llc}
\hline Vectors & Fusion Protein & Secretion Capacity \\
\hline pLacUV5 $^{\text {a }}$ & sSpAD-N ${ }^{\text {pro EDDIE-pep6His }}$ & $11.3 \mathrm{mg} / \mathrm{L}$ \\
\hline pLacUV5 & sSpAD-Gfpmut3.1 & $1.3 \mathrm{mg} / \mathrm{L}$ \\
\hline pLacUV5 & sSpAD-SOD & $16.4 \mathrm{mg} / \mathrm{L}$ \\
\hline
\end{tabular}

${ }^{a}$ pET30a plasmids with the lacUV5 promoter are named pLacUV5

periplasm without sSpAD subtracted from the amount of proteins secreted with the sSpAD tag. The corrected secretion capacities are given in Table 1 . The secretion yields for sSpAD-SOD $(16.4 \mathrm{mg} / \mathrm{L})$ and sSpAD-N ${ }^{\text {pro }} E D-$ DIE-pep6His $(11.3 \mathrm{mg} / \mathrm{L})$ were well above the reported yield for Sec mediated secretion [9]. However, in contrast to $\mathrm{N}^{\text {pro }}$ EDDIE-pep6His (not detectable in the periplamic fraction) and SOD (approximately 30 percent in the periplamic fraction), a major part of GFPmut3.1 was found in the periplasm, although the lack of the cytoplasmic chaperone GroEL indicated intact spheroplasts. Due to expression of GFPmut3.1 from a codonoptimized gene the protein level of GFPmut3.1 was rather high and increased even with the sSpAD tag. Therefore it was assumed, that these high expression levels provoked a passive diffusion of GFPmut3.1 into the periplasm.

\section{One step secretion and purification}

In order to exemplify the quick and easy purification of fusion proteins the periplasmic fraction of a $10 \mathrm{ml}$ shaking flask cultivation of sSpAD-SOD was purified. The sample was applied on an IgG column as described in Methods. After a single purification step $16.9 \mathrm{mg} / \mathrm{L}$ of the purified fusion protein could be obtained (Figure 4). This was in good agreement to the amount found by determination of the secretion capacity $(16,4 \mathrm{mg} / \mathrm{L})$.

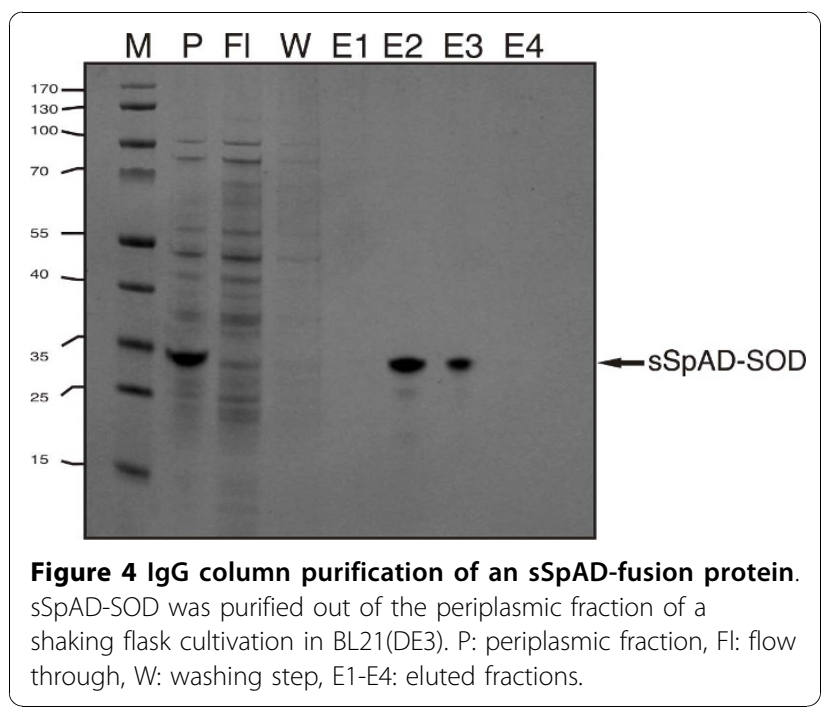

\section{Conclusions}

The main advantage of this system is the applicability for a variety of different proteins and the improved yield of soluble product. Especially for aggregate forming proteins this tag provides an alternative to common solubility tags such as GST and MBP. In terms of secretion of heterologous targets, a typical Sec signal sequence is often not sufficient to promote the transport across the inner cell membrane. The sSpAD tag tends to enhance the solubility of aggregate forming fusion partners, which results in an improved secretion of the target protein. This was exemplified by the autoprotease $\mathrm{N}^{\text {pro }}$, a cystein rich protein, which does not secrete with a single Sec signal peptide (data not shown). Furthermore, the tag facilitates a straight-forward one step purification of the target protein, which was shown by the purification of sSpAD-SOD. Since sSpAD was not processed during Sec mediated secretion a proteolytic cleavage of the tag is necessary. Screening of sSpAD with the SignalP 3.0 prediction tool did not result in the detection of an intrinsic secretion signal. With an overall length of $7 \mathrm{kD}$ the tag does not suit the classic Sec signal sequence. Therefore, it is proposed that SSpAD is not cleaved by the signal peptidase and possibly activates the SecA translocation by its conformation. Further dissection of sSpAD may identify an intrinsic secretion signal, which still facilitates an affinity-mediated purification.

\section{Methods}

All experiments were performed with Milli-Q ultrapure water (Millipore purification system). E. coli cells were cultivated in TY growth medium supplemented with $50 \mu \mathrm{g} / \mathrm{ml}$ kanamycin and/or $100 \mu \mathrm{g} / \mathrm{ml}$ ampicillin depending on the used plasmid. For recombinant plasmid isolation E. coli $\mathrm{K} 12 \mathrm{DH} 5 \alpha$ strain was used, whereas protein expression was performed in BL21(DE3), CM124 ( $\triangle$ SecE with SecE under control of the araBAD promoter placed on a plasmid) and MC4100-DADE $(\triangle \mathrm{tat} \mathrm{ABCDE})$.

Restriction enzymes, GoTaq ${ }^{\circ}$ DNA polymerase, including the PCR buffer, were obtained from Promega. Molecular mass standard used for SDS-PAGE, rapid DNA ligation kit, Pfu DNA polymerase and 10x $\mathrm{MgSO}_{4}$-PCR buffer were obtained from Fermentas. Tris-Glycine gels were purchased from Invitrogen. Protran BA 83 nitrocellulose membrane was obtained from Whatman. Mouse anti-GroEL monoclonal antibody was purchased from Stressgen Bioreagents, Goat anti-Mouse IgG (HRP conjugated) from Invitrogen, anti-Maltose Binding Protein (MBP) monoclonal antibody (HRP conjugated) and anti-Maltose Binding Protein (MBP) polyclonal antibody from New England Biolabs. Anti-N $\mathrm{N}^{\text {pro }}$ antibody was generated within the Austrian Center of 
Biopharmaceutical Technology at the BOKU Vienna. Syringe filters (pore size $0.45 \mu \mathrm{m}$ ) were from Sartorius, $10 \mathrm{kDa}$ molecular weight cut-off ultrafiltration devices (Centriprep Ultracel YM-10 tubes, series 8000 stirred cell including Ultracel YM-10 membranes) from Millipore. The $\mathrm{BCA}^{\mathrm{T}}$ protein assay kit was obtained from Pierce.

\section{Construction of expression plasmids}

The pET30a T7 promoter (T7p) was replaced by three alternative promoters e.g. T5, the artificial Tac promoter and the lacUV5 promoter resulting in the three vectors pT5, pTac and pLacUV5. Two oligonucleotides corresponding to the given promoter sequence with complementary bases, prom lacUV5 SphI F and prom lacUV5 XbaI R, were directly ligated into an SphI and XbaI digested pET30a plasmids. The Tac promoter, consisting of two oligonucleotides with 65 complementary bases, prom Tac SphI F and prom Tac XbaI R, was directly ligated into SphI and XbaI digested pET30a plasmids. The T5 promoter was generated by PCR with the given primers in Table 2 and subsequently cloned into SphI and XbaI digested pET30a plasmids.

Codon optimized GFPmut3.1 gene was amplified using the primers sGFP F NdeI start and sGFP R (Table 2 ). The amplified fragment was ligated into NdeI and SalI digested pLacUV5 vector, resulting in pLacUV5GFPmut3.1 plasmid.

The sSpAD signal sequence was codon optimized (sequence given in the appendix 1) and amplified using the primers pET30 sSpAD NdeI F and pET30 sSpAD NdeI R. Subsequently sSpAD was subcloned into the pLacUV5 GFPmut3.1 vector resulting in the pLacUV5sSpAD-GFPmut3.1.
The sSpAD-N ${ }^{\text {pro }}$-EDDIE-pep6His construct was generated by digestion with NdeI of the pET30 $\mathrm{N}^{\text {pro }}$ EDDIE-pep6His vector and subsequent ligation with the same insert generated for the pLacUV5-sSpADGFPmut3.1 construct. Subsequently the promoter of the pET30-sSpAD-N ${ }^{\text {pro }}$-EDDIE-pep6His construct was replaced with the promoter LacUV5.

The pLacUV5-sSpAD-SOD plasmid was generated in two steps. First sSpAD was amplified using the primers pET 30 sSpAD NdeI F and pLacUV5 sSpAD NheI $R$ and cloned into the pLacUV5 vector, which resulted in the pLacUV5 vector with an additional NheI restriction site. Subsequently the codon optimized SOD gene was amplified using the primers SOD NheI F and SOD SalI $\mathrm{R}$ (Table 2). The amplified fragment was ligated into the pLacUV5-sSpAD-Nhe vector, which was digested with NheI and SalI, resulting in pLacUV5 sSpAD-SOD plasmid. A list of the constructs is given in Table 3.

\section{Shake flask cultivation}

Expression plasmids were transformed into E. coli BL21 (DE3), subsequently a single colony was picked to inoculate overnight cultures. These cultures were diluted 1:20 with fresh TY-medium, supplemented with $0.5 \%$ glucose and grown to a density of $\mathrm{OD}_{600}: 1.0$ at $37^{\circ} \mathrm{C} /$ $225 \mathrm{rpm}$. Recombinant protein synthesis was induced adding $1 \mathrm{mM}$ IPTG. Expression with T5 promoter was carried out for $1 \mathrm{~h}$ at $37^{\circ} \mathrm{C} / 225 \mathrm{rpm}$ in BL21(DE3), BL21 (DE3) with $2 \mathrm{mM} \mathrm{NaN}_{3}$, BL21(DE3) $50 \mu \mathrm{M}$ CCCP. For the shake flask cultivation with $E$. coli DADE experimental procedure was similar to $E$. coli BL21(DE3) but without addition of glucose during cultivation.

In contrast overnight cultures of CM124 cells carrying pET30-Tac-promoter-plasmids were grown in the

Table 2 Oligonucleotides used in this study

\begin{tabular}{|c|c|}
\hline Primers & Sequences $\left(5^{\prime}-3^{\prime}\right)$ \\
\hline pET 30 sSpAD Ndel F & GCACGACATATGGCAGACGCACAACAGAATAAG \\
\hline pET 30 sSpAD Ndel R & TAGCAGCATATGTITTGGTGCCTGGAGTTC \\
\hline pLacUV5 sSpAD Nhel R & GCAAGCTAGCTITGGTGCCTGAGATTCGTTC \\
\hline SOD Nhel F & TAAAGCTAGCGCGGCAACAAAGGCCGTGTG \\
\hline SOD Sall R & AGTTGTCGAC TTGGGCGATCCCAATTACACC \\
\hline sGFP F Ndel & GGATCCACTCATATGAGCAAAGGCGAAG \\
\hline sGFP R & CGAGGTCGACTTATTATITATACAGTTCATC \\
\hline prom Tac Sphl F & 5'P-CGAGCTGTTGACAATTAATCATCGGCTCGTATAATGTGTGGAATTGTGAGCGGATAACAATTT \\
\hline prom Tac Xbal R & 5'P-CTAGAAATTGTTATCCGCTCACAATTCCACACATTATACGAGCCGATGATTAATTGTCAACAGCTCGCATG \\
\hline prom LacUV5 Sphl F & 5'P-CCCAGGCTITACACTTATGCTTCCGGCTCGTATAATGTGTGGAATTGTGAGCGGATAACAATIT \\
\hline prom LacUV5 Xbal R & 5'P-CTAGAAATTGTTATCCGCTCACAATTCCACACATTATACGAGCCGGAAGCATAAAGTGTAAAGCCTGGGCATG \\
\hline T5 Prom Sphl F & GGCGGCATGCGAAATCATAAAAAATTTAT \\
\hline T5 Prom Xbal R & TTCTAGATGTGTGAAATTGTTATCCGCT \\
\hline
\end{tabular}


Table 3 Plasmids and corresponding expression products

\begin{tabular}{|c|c|c|c|}
\hline Vectors $^{\mathrm{a}, \mathrm{b}, \mathrm{c}}$ & Gene cloned & Resulting vectors & Expression product \\
\hline pT5 b & sSpAD-N ${ }^{\text {pro }}$ EDDIE-pep6His & 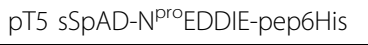 & 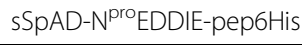 \\
\hline $\mathrm{pTac}^{\mathrm{c}}$ & 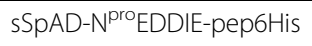 & 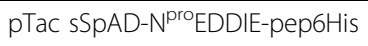 & sSpAD-N ${ }^{\text {pro EDDIE-pep6His }}$ \\
\hline $\operatorname{pLacUV5}^{\mathrm{a}}$ & sSpAD-Gfpmut3.1 & placUV5 sSpAD-Gfpmut3.1 & sSpAD-Gfpmut3.1 \\
\hline pLacUV5 & sSpAD-SOD & placUV5 sSpAD-SOD & sSpAD-SOD \\
\hline
\end{tabular}

${ }^{a}$ pET30a plasmids with the lacUV5 promoter are named pLacUV5

${ }^{b}$ pET30a plasmids with the T5 promoter are named pT5

c $\mathrm{pET} 30 \mathrm{a}$ plasmids with the Tac promoter are named pTac

presence of $0.2 \% \mathrm{~L}$-arabinose. These overnight cultures were diluted 1:20 and split. The divided cultures were grown with and without $0.2 \% \mathrm{~L}$-arabinose in parallel. Expression was induced at $\mathrm{OD}_{600}: 0.5$ with $1 \mathrm{mM}$ IPTG and the cultures were incubated for $1 \mathrm{~h}$ at $37^{\circ} \mathrm{C} /$ $225 \mathrm{rpm}$.

For the determination of the solubility and the secretion capacity overnight cultures of the host strain BL21 (DE3) carrying the pET30-LacUV5-promoter plasmids were diluted 1:20. Expression was induced at $\mathrm{OD}_{600}: 0.5$ with $1 \mathrm{mM}$ IPTG and the cultures were incubated for $2 \mathrm{~h}$ at $37^{\circ} \mathrm{C} / 225 \mathrm{rpm}$.

\section{Cell fractionation}

Isolation of the periplasm was performed at $24^{\circ} \mathrm{C}$ with a gentle osmotic shock procedure to minimize $E$. coli cell disruption during preparation (modified from [15]). After expression, a culture volume corresponding to 10 $\mathrm{ml}$ of $\mathrm{OD}_{600} 2.0$ was centrifuged at $3000 \mathrm{~g}$ for $10 \mathrm{~min}$. The pellet was completely suspended in one culture volume of osmolysis buffer (100 mM TRIS- $\mathrm{HCl} \mathrm{pH} 7.8$, $15.4 \%$ sucrose, $3 \mathrm{mM}$ EDTA) and incubated at $50 \mathrm{rpm}$ for $10 \mathrm{~min}$, followed by centrifugation. The supernatant was discarded and the pellet was resuspended in one culture volume water and incubated for $10 \mathrm{~min}$ at $50 \mathrm{rpm}$. Afterwards the suspension was centrifuged for $10 \mathrm{~min}$ at $3000 \mathrm{~g}$. The supernatant, containing periplasmic proteins, was decanted and filtrated (membrane, $0.45 \mu \mathrm{m}$ pore size). The spheroplasts were kept as a cellular fraction. For SDS-PAGE analysis periplasmic and cytoplasmic fraction samples were precipitated with TCA and all pellets solubilised in loading buffer $(62.5$ $\mathrm{mM}$ Tris- $\mathrm{HCl} \mathrm{pH} 6.8,10 \%$ glycerol, $2 \%$ SDS, $0.0025 \%$ bromophenol blue, $50 \mathrm{mM}$ DTT).

For the determination of the solubility the cells were taken up in a culture volume lysis buffer $(20 \mathrm{mM}$ $\mathrm{Na}_{2} \mathrm{HPO}_{4}$ pH 8.0, $75 \mathrm{mM} \mathrm{NaCl}, 5 \mathrm{mM}$ EDTA) and disrupted with a French press (American Instruments Co., Inc). Aliquots of the lysate were collected and centrifuged at $14000 \mathrm{rpm}$ for $15 \mathrm{~min}$. The supernatant contained soluble cytoplasmic protein, whereas the pellet represented the insoluble protein fraction. For SDS-
PAGE analysis the samples were precipitated with TCA and all pellets solubilized in loading buffer $(62.5 \mathrm{mM}$ Tris- $\mathrm{HCl} \mathrm{pH} 6.8,10 \%$ glycerol, $2 \%$ SDS, $0.0025 \%$ bromophenol blue, $50 \mathrm{mM}$ DTT).

\section{Concentration and purification of recombinant protein}

To the filtrated periplasm fraction $\mathrm{Na}_{2} \mathrm{HPO}_{4} / \mathrm{NaH}_{2} \mathrm{PO}_{4}$ and $\mathrm{NaCl}$ were added to a final concentration of $20 \mathrm{mM}$ $\mathrm{Na}_{2} \mathrm{HPO}_{4} / \mathrm{NaH}_{2} \mathrm{PO}_{4}, 500 \mathrm{mM} \mathrm{NaCl}, \mathrm{pH} 8.10 \mathrm{ml}$ of this solution were concentrated to $2 \mathrm{ml}$ with ultrafiltration devices. After removal of precipitated proteins by centrifugation, the supernatant was applied to a pre-equilibrated $500 \mu \mathrm{l}$ gravity flow IgG-NHS-Sepharose column and purified. The column was washed with $2.5 \mathrm{ml} 20$ $\mathrm{mM} \mathrm{Na}_{2} \mathrm{HPO}_{4} / \mathrm{NaH}_{2} \mathrm{PO}_{4} 500 \mathrm{mM} \mathrm{NaCl} \mathrm{pH} 8$ buffer. The recombinant protein was eluted with 5 column volumes of 0.2 glycine buffer $\mathrm{pH} 3$. The eluted fractions were pooled and the total concentration of the purified protein was quantified.

\section{Immuno blots}

Cellular integrity after expression of the recombinant proteins and subsequent osmotic shock treatment was surveyed by Immuno blot analysis with antibodies against the periplasmic Maltose binding protein MalE, and the cytoplasmic chaperone GroEL. Cell fraction samples were separated on 4-20\% Tris-Glycine gels and the proteins electrophoretically transferred onto nitrocellulose membranes. Incubation times of the antibodies were carried out according the instruction manuals.

\section{Protein quantification}

Quantification of the secretion capacity was carried out by densitometric analysis of target proteins in comparison to BSA standards on Coomassie stained gels. Gels were photographed and analyzed with AlphaEaseFC software (Alpha Innotech Corporation).

\section{Appendix 1}

\section{Accession numbers}

GFPmut3.1 [P42212]; SOD [BT008028.1]; Staphylococ-

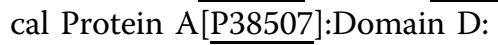




\author{
ADAQQNKFNKDQQSAFYEILNMPNLNEEQRNG- \\ FIQSLKDDPSQSTNVLGEAKKLNESQAPK N ${ }^{\text {pro EDDIE }}$ \\ [7] \\ DNA sequence sSpAD (codon optimized): \\ GCAGACGCACAACAGAATAAGTTTAACAAAGA \\ CCAGCAGAGCGCATTCTACGAAATTCTGAACAT \\ GCCGAATCTGAATGAGGAACAACGTAATGGCT \\ TTATTCAGTCTTTAAAAGACGACCCATCTCAGA \\ GCACCAACGTTCTGGGCGAAGCAAAGAAACTG \\ AACGAATCTCAGGCACCAAAA
}

\begin{abstract}
Abbreviations used
sSpAD: Staphylococcus aureus Protein A domain D expressed from a synthetic (codon optimized) gene; BL21(DE3): host strain; CCCP: carbonyl cyanide m-chlorophenylhydrazone; DADE: host strain derived from MC4100 $\triangle$ tatABCD, $\triangle$ tatE; DTT: dithiothreitol; EDTA: ethylene-diamine-tetra-acetic acid; GroEL: chaperone heat shock protein 60; GFP: Green fluorescent protein; IPTG: isopropyl- $\beta$-D-thiogalactopyranoside; MalE: maltose binding protein E; $\mathrm{NaN}_{3}$ : sodium-azide; PCR: polymerase chain reaction; SDS: sodium dodecyl sulphate; SOD: Human Superoxide Dismutase 1; TCA: trichloro acetic acid;
\end{abstract}

\section{Acknowledgements}

The present work was performed within the Austrian Center of Industrial Biotechnology (ACIB), a competence center funded by the Austrian Ministry of Economics and Labor, the federal states of Vienna and Tyrol and by its industrial partners Sandoz GmbH and Boehringer Ingelheim Austria GmbH. The TAT knock-out strain DADE was kindly provided by Dr. Tracy Palmer. The SecE knock-out strain CM124 Strain was kindly provided by Dr. JanWillem de Gier. Katherin Patsch edited the manuscript.

\section{Author details}

${ }^{1}$ Austrian Center of Industrial Biotechnology (ACIB), TU Graz, Petersgasse 14, A-8010 Graz, Austria. ${ }^{2}$ Institute of Biochemistry, University of Innsbruck, PeterMayr-Strasse 1a, A-6020 Innsbruck, Austria. ${ }^{3}$ Center for Molecular Biosciences (CMBI), University of Innsbruck, Peter-Mayr-Strasse 1a, A-6020 Innsbruck, Austria.

\section{Authors' contributions}

$\mathrm{TH}$ performed the experiments and wrote the manuscript. MP was involved in cloning the constructs and writing the manuscript. RS and BA were involved in the design of the experiments. All authors participated in editing the manuscript and all have read and approved the final version.

\section{Competing interests}

The authors declare that they have no competing interests.

Received: 26 August 2010 Accepted: 23 November 2010 Published: 23 November 2010

\section{References}

1. Jana S, Deb JK: Strategies for efficient production of heterologous proteins in Escherichia coli. Appl Microbiol Biotechnol 2005, 67(3):289-298.

2. Sorensen HP, Mortensen KK: Advanced genetic strategies for recombinant protein expression in Escherichia coli. J Biotechnol 2005, 115(2):113-128.

3. Swartz JR: Advances in Escherichia coli production of therapeutic proteins. Curr Opin Biotechnol 2001, 12(2):195-201.

4. Baneyx F: Recombinant protein expression in Escherichia coli. Curr Opin Biotechnol 1999, 10(5):411-421.

5. Sorensen HP, Mortensen KK: Soluble expression of recombinant proteins in the cytoplasm of Escherichia coli. Microb Cell Fact 2005, 4(1):1.

6. Vallejo LF, Rinas U: Strategies for the recovery of active proteins through refolding of bacterial inclusion body proteins. Microb Cell Fact 2004, 3(1):11.

7. Achmuller C, Kaar W, Ahrer K, Wechner P, Hahn R, Werther F, Schmidinger $\mathrm{H}$, Cserjan-Puschmann M, Clementschitsch F, Striedner $\mathrm{G}$, et al: $\mathrm{N}$ (pro) fusion technology to produce proteins with authentic $\mathrm{N}$ termini in E. coli. Nat Methods 2007, 4(12):1037-1043.
8. Belagaje RM, Reams SG, Ly SC, Prouty WF: Increased production of low molecular weight recombinant proteins in Escherichia coli. Protein Sci 1997, 6(9):1953-1962.

9. Mergulhao FJ, Summers DK, Monteiro GA: Recombinant protein secretion in Escherichia coli. Biotechnol Adv 2005, 23(3):177-202.

10. Georgiou G, Segatori L: Preparative expression of secreted proteins in bacteria: status report and future prospects. Curr Opin Biotechnol 2005, 16(5):538-545.

11. Kajava AV, Zolov SN, Kalinin $A E$, Nesmeyanova MA: The net charge of the first 18 residues of the mature sequence affects protein translocation across the cytoplasmic membrane of gram-negative bacteria. J Bacterio/ 2000, 182(8):2163-2169.

12. Mallik I, Smith MA, Flower AM: Recognition of secretory proteins in Escherichia coli requires signals in addition to the signal sequence and slow folding. BMC Microbiol 2002, 2:32.

13. Abrahmsen L, Moks T, Nilsson B, Hellman U, Uhlen M: Analysis of signals for secretion in the staphylococcal protein A gene. EMBO J 1985, 4(13B):3901-3906.

14. Nygren PA, Stahl S, Uhlen M: Engineering proteins to facilitate bioprocessing. Trends Biotechnol 1994, 12(5):184-188.

15. Kaar W, Ahrer K, Durauer A, Greinstetter S, Sprinzl W, Wechner P, Clementschitsch F, Bayer K, Achmuller C, Auer B, et al: Refolding of Npro fusion proteins. Biotechnol Bioeng 2009, 104(4):774-784.

16. Paal M, Heel T, Schneider R, Auer B: A novel Ecotin-Ubiquitin-Tag (ECUT) for efficient, soluble peptide production in the periplasm of Escherichia coli. Microb Cell Fact 2009, 8:7.

17. Natale $P$, Bruser T, Driessen AJ: Sec- and Tat-mediated protein secretion across the bacterial cytoplasmic membrane-distinct translocases and mechanisms. Biochim Biophys Acta 2008, 1778(9):1735-1756.

18. Driessen AJ: Bacterial protein translocation: kinetic and thermodynamic role of ATP and the protonmotive force. Trends Biochem Sci 1992, 17(6):219-223.

19. Santini CL, Ize B, Chanal A, Muller M, Giordano G, Wu LF: A novel secindependent periplasmic protein translocation pathway in Escherichia coli. EMBO J 1998, 17(1):101-112.

20. Wexler M, Sargent F, Jack RL, Stanley NR, Bogsch EG, Robinson C, Berks BC, Palmer T: TatD is a cytoplasmic protein with DNase activity. No requirement for TatD family proteins in sec-independent protein export. J Biol Chem 2000, 275(22):16717-16722.

21. Traxler B, Murphy $C$ : Insertion of the polytopic membrane protein MalF is dependent on the bacterial secretion machinery. J Biol Chem 1996, 271(21):12394-12400.

22. Bendtsen JD, Kiemer L, Fausboll A, Brunak S: Non-classical protein secretion in bacteria. BMC Microbiol 2005, 5:58.

23. Jeffery $\mathrm{CJ}$ : Moonlighting proteins: old proteins learning new tricks. Trends Genet 2003, 19(8):415-417.

doi:10.1186/1475-2859-9-92

Cite this article as: Heel et al:: Dissection of an old protein reveals a novel application: domain D of Staphylococcus aureus Protein A (sSpAD) as a secretion - tag. Microbial Cell Factories 2010 9:92.

\section{Submit your next manuscript to BioMed Central and take full advantage of:}

- Convenient online submission

- Thorough peer review

- No space constraints or color figure charges

- Immediate publication on acceptance

- Inclusion in PubMed, CAS, Scopus and Google Scholar

- Research which is freely available for redistribution

Submit your manuscript at www.biomedcentral.com/submit
C Biomed Central 